\title{
Correlation between corneal stromal demarcation line depth and topographic outcomes after two pulsed-light-accelerated crosslinking protocols
}

This article was published in the following Dove Press journal: Clinical Ophthalmology

\author{
Julio C Hernandez- \\ Camarena iD \\ Enrique $O$ Graue- \\ Hernandez 2 \\ Denise Loya-García' \\ Raul E Ruiz-Lozano' \\ Jorge E Valdez-García' \\ 'Tecnologico de Monterrey, Escuela de \\ Medicina y Ciencias de la Salud, \\ Monterrey, NL 647/0, Mexico; \\ ${ }^{2}$ Department of Cornea and Refractive \\ Surgery, Institute of Ophthalmology \\ Conde de Valenciana, Mexico City, \\ Mexico
}

Purpose: To report the visual and topographic outcomes of two pulsed-light-accelerated CXL (A-CXL) protocols at a 12-month follow-up and their correlation with the corneal stromal demarcation line (DL) depth.

Patients and methods: Retrospective comparative cohort of patients with documented progressive keratoconus were included. Two epi-off pulsed-light [1s on-1s off] A-CXL protocols were compared: irradiance $30 * 8$ and $45 * 5: 20$ (fluence $7.2 \mathrm{~J} / \mathrm{cm}^{2}$ ). UDVA, CDVA, spherical equivalent (SE), topographic astigmatism, $\mathrm{K}_{\min }, \mathrm{K}_{\mathrm{max}}, \mathrm{K}_{\mathrm{m}}$, central corneal thickness (CCT), thinnest pachymetry (TCT) and endothelial cell density (ECD) were measured preoperatively and months 1, 3,6 and 12 postoperative. Corneal DL was measured 1 month postoperatively using anterior segment optical coherence tomography.

Results: Fifty eyes (27 patients): 22 eyes in group A-CXL $(30 * 8), 28$ eyes in group A-CXL (45*5:20). Mean age (years) was $19.04 \pm 4.71$ and $20.32 \pm 4.57$. DL depth $(\mu \mathrm{m})$ at month 1 was $200.63 \pm 10.01 \mu \mathrm{m}$ and $184.53 \pm 19.68 \mu \mathrm{m}$ for group A-CXL $\left(30^{*} 8\right)$ and group A-CXL $\left(45^{*} 5: 20\right)$, respectively $(p<0.001)$. Significant improvement in CDVA, topographic astigmatism, $\mathrm{K}_{\min }, \mathrm{K}_{\max }$ and $\mathrm{K}_{\mathrm{m}}$ was observed in both groups (no significant difference between groups) and no significant changes were observed in CCT, TCT and ECD with regard to baseline. Over $85 \%$ of the eyes in both protocols achieved stabilization or improvement in maximum $\mathrm{K}$ at the end of the follow-up. No significant correlations between DL and any visual or topographic outcomes were observed at 12 months.

Conclusion: No correlation between DL depth and visual or topographic outcomes was observed on either protocol. Although significant improvement on CDVA, topographic astigmatism, $\mathrm{K}_{\min }, \mathrm{K}_{\max }$ and $\mathrm{K}_{\mathrm{m}}$ was observed in both groups at 12 months, further research is needed to assure safety and effectiveness at stabilizing keratoconus progression.

Keywords: keratoconus, accelerated corneal crosslinking, corneal stromal demarcation line, optical coherence tomography, topographic stabilization

\section{Introduction}

Corneal crosslinking (CXL) has been used for the treatment of progressive keratoconus for more than a decade, halting the progression and stabilizing vision and corneal curvature in most cases. ${ }^{1-4}$ The Dresden protocol, consisting of a $3 \mathrm{~mW} / \mathrm{cm}^{2}$ irradiance during 30 mins and the use of riboflavin as a photosensitizer, has been considered as the standard CXL treatment as extensive evidence has demonstrated its safety and efficacy on improving clinical and topographical outcomes. ${ }^{1,2,5,6}$ Novel accelerated CXL modalities have allowed shorter treatment periods through higher irradiance levels, keeping a constant total energy dose (Bunsen-Roscoe law of
Correspondence: Julio C HernandezCamarena

Cornea and Refractive Surgery Service, Zambrano-Hellion Hospital, Batallon de San Patricio II2, Ist Floor East, Real de San Agustín, San Pedro Garza Garcia,

Nuevo Leon 66278 , Mexico

Tel +52 I 8188880550

Fax +52 । 8188880555

Email dr.juliohernandez@medicos.tecsalud. $\mathrm{mx}$ 
reciprocity). ${ }^{7-9}$ Moreover, the introduction of pulsed UVA light CXL methods that permit a better oxygen diffusion to the corneal stroma during the irradiation period has enhanced the efficacy of high fluence (accelerated) CXL treatment modalities. ${ }^{10-12}$

Regardless of the CXL modality treatment, the corneal stromal demarcation line (DL) central depth has been continuously proposed as a feasible predictor for clinical and topographic efficacy and as an objective measurement of stromal collagen fiber crosslinking. ${ }^{13-16}$ Higher fluence irradiation and continuous UV light modalities have been associated with a more anterior (superficial) DL and with a lessen effect of corneal CXL on visual and topographic outcomes when compared with the Dresden protocol and pulsed UV light treatment modalities. ${ }^{4,711,17}$ However, there is limited evidence on the efficacy of pulsed accelerated CXL protocols to halt or stabilize keratoconus progression and on the correlation of corneal DL with the visual and topographic outcomes of these CXL modalities. We report the visual and topographic results of two different pulsed-light-accelerated CXL protocols $\left(30 \mathrm{~mW} / \mathrm{cm}^{2}\right.$ and $45 \mathrm{~mW} / \mathrm{cm}^{2}$ ) at one-year follow-up and compare and correlate the DL of the two protocols with the functional outcomes.

\section{Materials and methods}

Eyes of patients with diagnosis of progressive keratoconus who underwent corneal accelerated pulsed-light [1 secondon, 1 second-off] CXL at $30 \mathrm{~mW} / \mathrm{cm}^{2}$ and $45 \mathrm{~mW} / \mathrm{cm}^{2}$ at the Hospital Zambrano-Hellion Tecnologico de Monterrey, San Pedro Garza García, Nuevo Leon, Mexico, between May 2017 and August 2018 were enrolled in this retrospective comparative cohort. The study was performed in accordance with the tenets of the Declaration of Helsinki and proper ethics approval was obtained from the local ethics committee (Ethics and Research Committee of the School of Medicine and Health Sciences, Tecnologico de Monterrey. National Bioethics Commission registration number 13CEI190390139). All enrolled patients or patients' legal tutors were informed about the benefits and risks of the procedure and provided written informed consent.

Inclusion criteria for corneal CXL were documented progression of keratoconus, defined as 1 or more of the following changes: change of $\geq 1 \mathrm{D}$ in the maximum anterior corneal curvature $\left(\mathrm{K}_{\max }\right)$, a thinning of more than 10 $\mu \mathrm{m}$ in the minimal pachymetry observed in 2 consecutive tomography maps or a $0.50 \mathrm{D}$ increase in spherical equivalent (SE) within 12 months. ${ }^{18}$ Patients with corneal thickness of $\geq 350 \mu \mathrm{m}$ were included only if intra-operative pachymetry $>400 \mu \mathrm{m}$ was achieved using hypotonic riboflavin solution. Exclusion criteria to receive CXL treatment were corneal apical scarring, concomitant ocular infection (including history of herpes keratitis), history of connective tissue diseases or systemic medications likely to affect corneal epithelization, pregnancy and lactation during the study period.

A full ophthalmological examination was performed to all patients before CXL treatment including uncorrected (UDVA) and corrected (CDVA) distance visual acuities, subjective refraction (sphere and cylinder) and SE, corneal topography including maximum $\left(\mathrm{K}_{\max }\right)$ and minimum $\left(\mathrm{K}_{\mathrm{min}}\right)$ and mean $\left(\mathrm{K}_{\mathrm{m}}\right)$ keratometries, topographic astigmatism (Ast) and minimal thinnest pachymetry (TCT) and central corneal thickness (CCT) (Pentacam HR, Oculus, Wetzlar, Germany). All measurements were performed at baseline, month 1, 3, 6 and 12 postoperative. Endothelial cell density (ECD) was measured at baseline, month 3, 6 and 12 postoperative (Cell Check SL, Konan Medical USA Inc, Irvine, CA).

Anterior segment OCT (Optovue Inc, Freemont, CA, USA) was performed at month 1 postoperatively; the corneal stromal DL was identified centrally on the horizontal meridian and measured with the flap tool provided by the manufacturer as the distance from corneal epithelium to the hyperreflective DL. Two independent examiners (D.L.G, J.C.H.C.) measured the central depth of the DL. Postoperative stabilization of keratoconus was defined as a change in maximum $\mathrm{K}$ of no more than $\pm 1.00 \mathrm{D} .^{19}$

\section{Surgical technique}

Corneal CXL was performed under sterile conditions. After topical anesthesia with tetracaine clorhidrate $0.5 \%$ (Ponti ofteno $^{\mathrm{TM}}$, Sophia Labs, Jalisco, Mexico), the central $9.0 \mathrm{~mm}$ of the corneal epithelium was mechanically debrided with a blunt spatula. After epithelium removal, $0.1 \%$ riboflavin in a hydroxy-propyl methylcellulose solution (Vibex Rapid, Avedro, Inc.) was applied every 2 mins during 10 mins into the corneal stromal bed. At the end of the riboflavin soak, the solution was rinsed from the ocular surface using balanced salt solution and central ultrasound pachymetry was performed (Accutome AccuPach V, Malvern, PA, USA). In patients with CCT $<400 \mu \mathrm{m}$, hypotonic riboflavin $0.1 \%$ solution (MedioCROSS H, Avedro, Inc) was instilled every $15 \mathrm{~s}$ until CCT was $\geq 400 \mu \mathrm{m}$. The corneal stroma was then 
exposed to pulsed [ $1 \mathrm{~s}$ on $-1 \mathrm{~s}$ off] UVA irradiation (365 nm light) using the Avedro KXL System (Avedro Inc., Waltham, Massachusetts, US) with a $30 \mathrm{~mW} / \mathrm{cm}^{2}$ irradiance for 8 mins [30*8] or a $45 \mathrm{~mW} / \mathrm{cm}^{2}$ irradiance during 5:20 mins [45*5:20]. A therapeutic soft contact lens (Purevision 2, Bausch \& Lomb, Inc., Rochester, NY) was applied at the end of the procedure and left in place until complete epithelial healing. Postoperative medications included moxifloxacin $0.5 \%$ (Vigamoxi; Alcon Laboratories, Inc., Fort Worth, TX) eye drops 4 times a day for 10 days and loteprednol etabonate $0.5 \%$ (Loterex $0.5 \%$, Bausch \& Lomb, Inc., Rochester, NY) 4 times a day in a tapering dose for 1 month.

\section{Statistical analysis}

Statistical analysis was performed using SPSS software version 23.0 (SPSS Inc.). To explore normal distribution, a Shapiro-Wilk test was performed. Continuous variables are expressed in mean $\pm \mathrm{SD}$. All variables showed normal distribution $(p>0.05)$; hence, chi-square test and paired $t$ test was used to compare mean differences in different follow-up periods with the baseline measurements within-groups and two sample $t$-test was used to compare means between the two A-CXL modalities $(30 * 8$ vs $45^{*} 5: 20$ ). Pearson correlation coefficient ( $r$ ) and multiple linear regression were used to determine the correlations and variability of the visual and topographic outcomes with the independent variables. A $p$-value of $<0.05$ was considered statistically significant.

\section{Results}

A total of 27 patients (50 eyes) with a 12-month follow-up posterior to pulsed-light A-CXL were included. Mean age was $19.76 \pm 4.63$ years (rank 13-28 years), 16 patients $(59 \%)$ were female. Twenty-two eyes were treated with the pulsed A-CXL $(30 * 8)$ protocol while 28 eyes were treated with the A-CXL $(45 * 5: 20)$, protocol. Mean age was $19.04 \pm 4.71$ years for A-CXL $(30 * 8)$ and $20.32 \pm 4.57$ years for A-CXL $\left(45^{*} 5: 20\right)$, no statistically significant difference was observed in baseline UDVA, CDVA, MRSE, Ast, $\mathrm{K}_{\min }, \mathrm{K}_{\max }, \mathrm{K}_{\mathrm{m}}, \mathrm{CCT}$, TCT and ECD $(p>0.05)$ (Table 1 shows demographic and baseline measurements).

All eyes exhibited the appearance of a corneal stromal DL 1 month postoperatively. Mean central DL depths were $200.63 \pm 10.01 \mu \mathrm{m}$ and $184.53 \pm 19.68 \mu \mathrm{m}$ for A-CXL $(30 * 8)$ and A-CXL $(45 * 5: 20)$, respectively $(p<0.001)$ (Figure 1). A non-significant improvement in UDVA was
Table I Baseline demographic, clinical and topographic parameters for two pulsed-light-accelerated CXL protocols

\begin{tabular}{|c|c|c|c|}
\hline & A-CXL $(30 * 8)$ & $\begin{array}{l}\text { A-CXL } \\
(45 * 5: 20)\end{array}$ & $p$-value \\
\hline $\mathrm{N}$ (patients) & 12 & 15 & \\
\hline$N$ (eyes) & 22 & 28 & \\
\hline Age (years) & $|9.04 \pm 4.7|$ & $20.32 \pm 4.57$ & 0.338 \\
\hline Gender & $9 F(75 \%)$ & $7 F(46 \%)$ & 0.165 \\
\hline AK Stage & 2.09 (Med 2) & $2.36($ Med 2) & 0.345 \\
\hline UDVA (LogMAR) & $1.15 \pm 0.55$ & $0.95 \pm 0.58$ & 0.135 \\
\hline CDVA (LogMAR) & $0.11 \pm 0.11$ & $0.08 \pm 0.08$ & 0.162 \\
\hline SE (D) & $-6.88 \pm 4.79$ & $-7.6| \pm 5.7|$ & 0.635 \\
\hline $\mathrm{K}_{\min }(\mathrm{D})$ & $49.42 \pm 6.74$ & $48.64 \pm 5.12$ & 0.644 \\
\hline $\mathrm{K}_{\max }(\mathrm{D})$ & $53.54 \pm 7.20$ & $54.80 \pm 6.66$ & 0.897 \\
\hline $\mathrm{K}_{\mathrm{m}}(\mathrm{D})$ & $51.48 \pm 6.71$ & $51.22 \pm 5.74$ & 0.882 \\
\hline Astigmatism (D) & $4.8 I \pm 2.60$ & $5.16 \pm 3.04$ & 0.672 \\
\hline ECD (cells $\left./ \mathrm{mm}^{2}\right)$ & $2594.18 \pm 368.28$ & $2552.25 \pm 340.60$ & $0.78 I$ \\
\hline CCT $(\mu \mathrm{m})$ & $471.86 \pm 62.29$ & $466.96 \pm 49.78$ & 0.758 \\
\hline TCT $(\mu \mathrm{m})$ & $453.27 \pm 68.19$ & 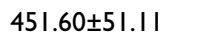 & 0.994 \\
\hline
\end{tabular}

Notes: None of the measurements was significantly different when comparing between groups [A-CXL $(30 * 8)$ and A-CXL $(45 * 5: 20)]$ (unpaired two sample $t$ test, normal distribution assessed with Shapiro-Wilk test).

Abbreviations: AK Stage, Amsler-Krumeich keratoconus stage; UDVA, uncorrected distance visual acuity; CDVA, corrected distance visual acuity; SE, spherical equivalent; Astigmatism, topographic astigmatism; $\mathrm{K}_{\min }$, minimum keratometry; $\mathrm{K}_{\text {max }}$, maximum keratometry; $\mathrm{K}_{\mathrm{m}}$, mean keratometry; $\mathrm{CCT}$, central corneal thickness; TCT, thinnest corneal thickness; ECD, endothelial cell density (central).

observed in group A-CXL $(30 * 8)$ during the follow-up and until month 12 postoperative. Likewise, group A-CXL (45*5:20) showed a similar performance in UDVA over time, but a statistically significant improvement was observed from month $6(p=0.017)$ until the end of the follow-up ( $p=0.007$, month 12 postoperative). A statistically significant improvement in CDVA compared with baseline measurements was observed at month 12 in both accelerated pulsed CXL groups (Table 2). No statistically significant differences were observed in UDVA or CDVA when comparing between groups through the follow-up period.

A non-significant decrease in SE was observed for both groups until month 3; in months 6 and 12 postoperative, a significant decrease with regard to baseline SE was observed (Table 2). Likewise, topographic astigmatism, $\mathrm{K}_{\min }, \mathrm{K}_{\max }$ and $\mathrm{K}_{\mathrm{m}}$ had a similar non-significant decrease until month 6 and 12 postoperative, when a significant difference (decrease) with regard to baseline measurements was observed. A significant decrease in CCT and TCT was observed for both groups in month 1 postoperative ( $p<0.001$ for both groups), observing a consecutive return to baseline values in corneal thickness toward month 12 postoperative (Table 2). No significant changes 


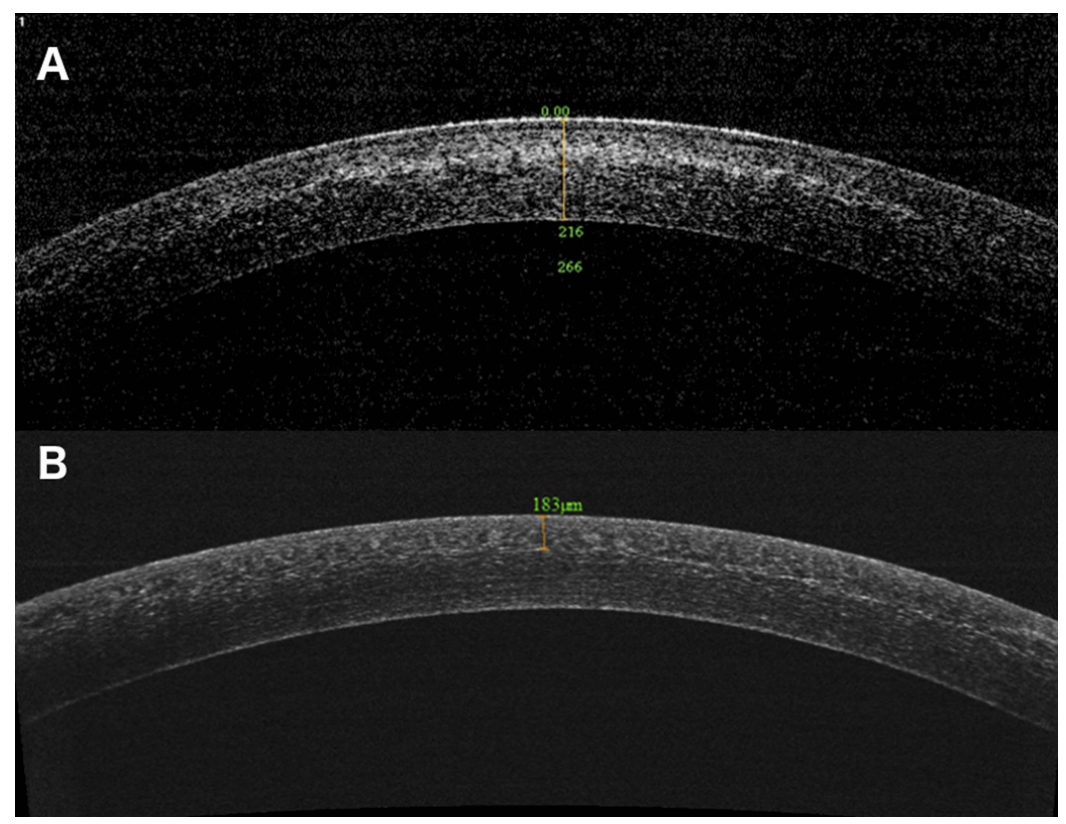

Figure I AS-OCT of corneal stromal demarcation line at postoperative month I in after pulsed-light-accelerated CXL with $30 \mathrm{~mW} / \mathrm{cm}^{2}(\mathbf{A})$ and $45 \mathrm{~mW} / \mathrm{cm}^{2}(\mathbf{B})$ irradiances. Abbreviation: CXL, corneal crosslinking.

were observed in ECD during the follow-up with regard to baseline measurements and none of the measurements was significantly different $(p>0.05)$ when comparing between groups [A-CXL $(30 * 8)$ vs A-CXL $(45 * 5: 20)$ ]. Table 3 summarizes the postoperative change $(\Delta)$ in visual acuity (UDVA, CDVA), SE, topography $\left(\mathrm{K}_{\min }, \mathrm{K}_{\max }, \mathrm{K}_{\mathrm{m}}\right)$ and pachymetry (CCT, TCT) from baseline values at 12 months.

No statistically significant correlation was observed between DL depth and changes in topographic astigmatism ( $\Delta$ Astigmatism, $\mathrm{r}=-0.197, p=0.218), \mathrm{K}_{\min }\left(\Delta \mathrm{K}_{\min }\right.$, $\mathrm{r}=0.215, p=0.177), \mathrm{K}_{\max }\left(\Delta \mathrm{K}_{\max }, \mathrm{r}=0.026, p=0.872\right)$ or $\mathrm{K}_{\mathrm{m}}\left(\Delta \mathrm{K}_{\mathrm{m}}, \mathrm{r}=0.123, p=0.444\right)$ throughout the follow-up. Also, no significant correlations were observed between DL at month 1 postoperative and $\triangle$ UDVA, $\triangle$ CDVA or $\Delta \mathrm{SE}$. Multiple linear regression analysis displayed baseline CCT as the most relevant factor to explain the variability of $\Delta \mathrm{K}_{\min } \quad\left(R_{\text {adjusted }}^{2}=0.203, \beta=0.351, p=0.024\right)$, $\Delta \mathrm{K}_{\max } \quad\left(R_{\text {adjusted }}^{2}=0.211, \beta=0.324, p=0.046\right)$ and $\Delta \mathrm{K}_{\mathrm{m}}$ $\left(R_{\text {adjusted }}^{2}=0.205, \beta=0.377, p=0.016\right)$ at 12 months follow-up in both groups (Figure 2). Nineteen eyes (86.5\%) in the A-CXL (30*8) group and 27 eyes (97\%) in the ACXL $\left(45^{*} 5: 20\right)$ group had regression ( $>1 \mathrm{D}$ decrease in $\mathrm{K}_{\max }$ in 12 months) or stabilization (no more than 1D change in $\mathrm{K}_{\max }$ at 12 months). Three eyes $(13.5 \%)$ in the A-CXL $(30 * 8)$ and one eye $(3 \%)$ in the A-CXL $(45 * 5: 20)$ group had progression $\left(>1 \mathrm{D}\right.$ increase in $\mathrm{K}_{\max }$ in 12 months). The distribution of regression, stabilization and progression was not different between the two different pulsed accelerated CXL protocols $\left(\chi^{2}=5.070, p=0.07\right)$ (Table 4).

\section{Discussion}

The corneal stromal DL is thought to be a direct marker of the effectiveness of CXL on the treated stroma; however, its precise pathophysiology and possible influence on the clinical and topographic outcomes is still indefinite. ${ }^{13,20}$ Evidence has shown that accelerated $\left(>3 \mathrm{~mW} / \mathrm{cm}^{2}\right)$ and continuous-light CXL protocols tend to form a more anterior or superficial corneal demarcation stromal line when compared with the original Dresden protocol and with pulsed-light treatments, respectively, associating a shallower DL with a reduced improvement on the functional and corneal topographic profile. ${ }^{4,11,12,21,22}$ We report the visual and topographic outcomes of two pulsed-light [1-1] accelerated CXL protocols [A-CXL (30*8) vs A-CXL $(45 * 5: 20)]$ at a 12 -month follow-up and their correlation with the DL depth.

We observed a significant difference in DL depth between protocols, with a depth of $200.63 \pm 10.01 \mu \mathrm{m}$ and $184.53 \pm 19.68$ $\mu \mathrm{m}$ for groups A-CXL $(30 * 8)$ and A-CXL (45*5:20), respectively. This in accordance with the reports by Mazzotta et al ${ }^{10}$ (198.43 $\mu \mathrm{m}$, range $188-212 \mu \mathrm{m})$, Shetty et $\mathrm{al}^{17}(201 \pm 82 \mu \mathrm{m}$, continuous-light CXL), Peyman et $\mathrm{al}^{23}(201.11 \pm 27.76 \mu \mathrm{m})$, 


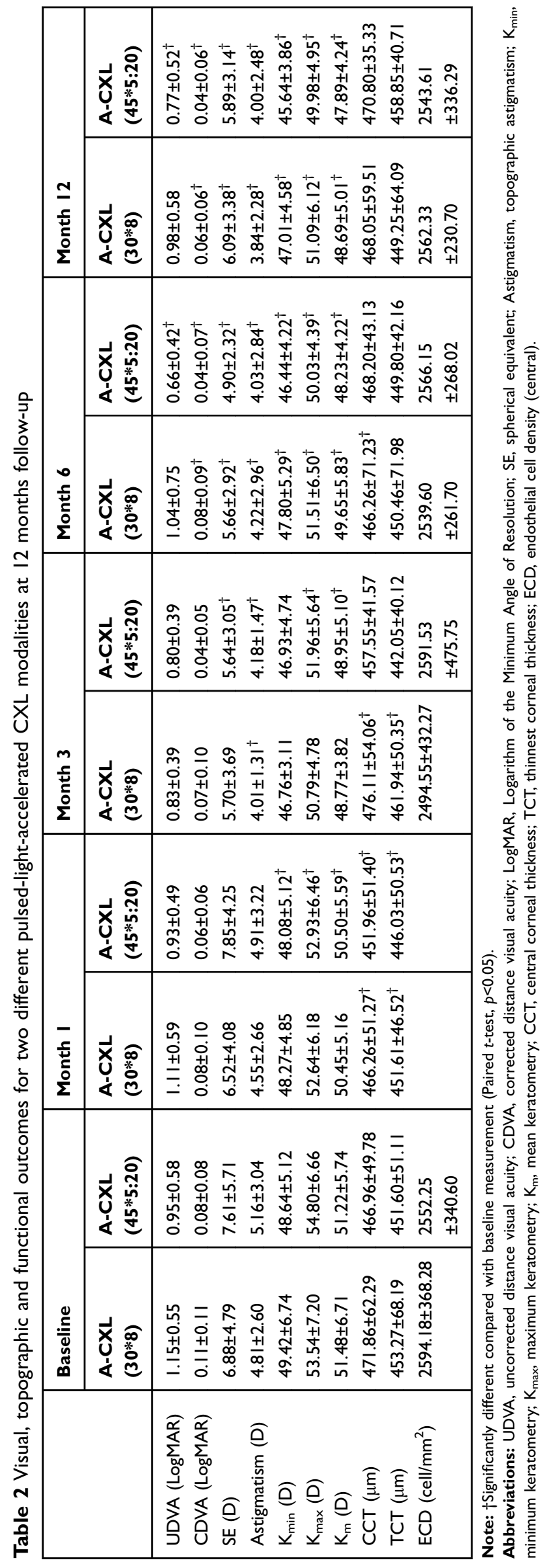

Table 3 Postoperative change in visual acuity, refractive error and topography at 12 months

\begin{tabular}{|l|l|l|}
\hline & A-CXL (30*8) & A-CXL (45*5:20) \\
\hline$\Delta$ UDVA (LogMAR) & $-0.22 \pm 0.51$ & $-0.25 \pm 0.39$ \\
$\Delta$ CDVA (LogMAR) & $-0.06 \pm 0.11^{\dagger}$ & $-0.04 \pm 0.05^{\dagger}$ \\
$\Delta$ SE (D) & $1.04 \pm 2.91^{\dagger}$ & $2.04 \pm 3.67^{\dagger}$ \\
$\Delta$ Astigmatism (D) & $-1.48 \pm 2.78^{\dagger}$ & $-0.81 \pm 2.07^{\dagger}$ \\
$\Delta \mathrm{K}_{\min }(\mathrm{D})$ & $-1.94 \pm 3.0 \mathrm{I}^{\dagger}$ & $-2.08 \pm 2.76^{\dagger}$ \\
$\Delta \mathrm{K}_{\max }(\mathrm{D})$ & $-2.98 \pm 3.09^{\dagger}$ & $-2.99 \pm 2.98^{\dagger}$ \\
$\Delta \mathrm{K}_{\mathrm{m}}(\mathrm{D})$ & $-2.68 \pm 2.72^{\dagger}$ & $-2.54 \pm 2.21^{\dagger}$ \\
$\Delta \mathrm{CCT}(\mu \mathrm{m})$ & $-6.25 \pm 17.63$ & $-7.30 \pm 18.94$ \\
$\Delta \mathrm{TCT}(\mu \mathrm{m})$ & $-6.60 \pm 20.74$ & $-6.14 \pm 22.05$ \\
\hline
\end{tabular}

Notes: $\nmid$ Significantly different compared with baseline measurement (Paired $t$-test, $p<0.05)$; None of the measurements was significantly different when comparing between groups [A-CXL $(30 * 8)$ and A-CXL $(45 * 5: 20)]$ through the follow-up period (unpaired two sample $t$-test).

Abbreviations: $\mathrm{SE}$, spherical equivalent; $\mathrm{K}_{\min }$, minimum keratometry; $\mathrm{K}_{\max }$, $\max -$ imum keratometry; $\mathrm{K}_{\mathrm{m}}$, mean keratometry; $\mathrm{CCT}$, central corneal thickness; $\mathrm{ECD}$, endothelial cell density (central).

Jiang et $\mathrm{al}^{24}(201.64 \pm 27.72 \mu \mathrm{m})$ and Moramarco et $\mathrm{al}^{11}(213$ $\pm 47.38 \mu \mathrm{m}$ ) for the $30 \mathrm{~mW} / \mathrm{cm}^{2}$ group, but shallower than that reported by Mariko $\mathrm{Mita}^{25}(\sim 300 \mu \mathrm{m}$, continuous-light CXL) and deeper than that reported by Toker et $\mathrm{al}^{4}(166 \pm 22 \mu \mathrm{m})$. To our knowledge, there is only one study by Zhang et $\mathrm{al}^{26}$ reporting the use of pulsed-light CXL with $45 \mathrm{~mW} / \mathrm{cm}^{2}$ irradiance; however, they did not observe a DL through anterior segment OCT, attributing this to the epithelial permeability to riboflavin since it was an epithelium-on CXL protocol. With regard to this, our data as well as previous clinical evidence, ${ }^{4,17}$ suggest that higher irradiance CXL protocols are associated with a shallower DL, as it was with the $45 \mathrm{~mW} / \mathrm{cm}^{2}$ irradiance group compared with the lower irradiance group (30 $\mathrm{mW} / \mathrm{cm}^{2}$ ).

Similar to Pircher et a ${ }^{27}$ who described the findings on a continuous-light-accelerated CXL protocol $\left(9 \mathrm{~mW} / \mathrm{cm}^{2}\right)$, our study did not observe a statistically significant correlation between the stromal central depth of the CDL and any topographic or visual outcome. On the other hand, $\mathrm{Ng}$ et al described a correlation between the deeper CDL on conventional CXL ( $3 \mathrm{~mW} / \mathrm{cm}^{2}$ protocol) and the change in mean keratometric readings $(\mathrm{r}=-0.432, \quad p=0.045){ }^{7}$ Likewise, Toker et $\mathrm{al}^{4}$ observed a moderate correlation between the DL depth and flattening in the keratometric values ( $\mathrm{K} 1, \mathrm{~K} 2$, mean $\mathrm{K}$ and maximum $\mathrm{K}$ ) and improvement in UDVA and CDVA for different accelerated CXL protocols after 12 months. Cumulative evidence with regard to the effectiveness of accelerated protocols to stabilize and/ or improve keratoconus seems to favor conventional and low irradiance CXL protocols, since deeper DL have been correlated in some studies with an increasing flattening 

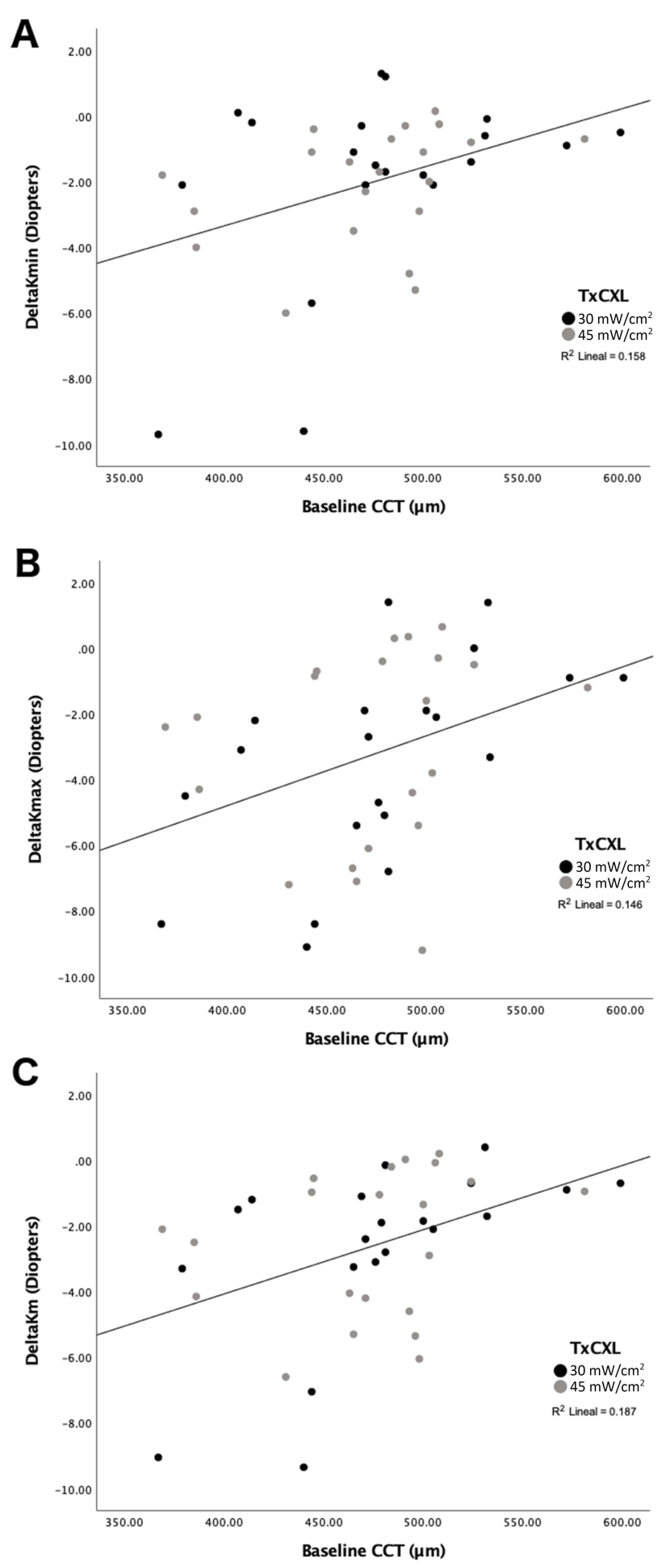

Figure 2 Pearson's correlation between baseline CCT and (A) $\Delta K_{\min }(r=0.38 I$, $p=0.014)$, (B) $\Delta \mathrm{K}_{\max }(r=0.473, p=0.002)$ and $(\mathbf{C}) \Delta \mathrm{K}_{\mathrm{m}}(\mathrm{r}=0.5 \mathrm{I} 7, p=0.00 \mathrm{I})$.

Abbreviation: CCT, central corneal thickness.

effect and improved clinical outcomes. ${ }^{4,7,17,24}$ However, the results in this and other similar studies ${ }^{10,11,24,26}$ suggest that pulsed-light-accelerated CXL protocols are effective in stabilizing and improving topographic and functional
Table 4 Percentage of regression ( $>$ ID decrease in $K_{\max }$ in 12 months), stabilization (no more than ID change in $K_{\max }$ ) and progression (>ID increase in $K_{\max }$ in 12 months) of keratoconus

\begin{tabular}{|l|l|l|l|}
\hline \multirow{2}{*}{ Group } & \multicolumn{3}{l|}{ Number (\%) } \\
\cline { 2 - 4 } & Regression & Stabilization & Progression \\
\hline A-CXL $(30 * 8)$ & $16(73)$ & $3(13.5)$ & $3(13.5)$ \\
A-CXL $(45 * 5: 20)$ & $19(68)$ & $7(25)$ & $2(7)$ \\
\hline
\end{tabular}

Note: No statistical difference between groups was observed (chi-square test). Abbreviation: A-CXL, accelerated crosslinking.

outcomes in eyes with progressive keratoconus despite the depth of the DL. In our series, over $85 \%$ of the eyes in both protocols achieved stabilization or improvement in maximum $\mathrm{K}$ at 12 months compared to baseline.

In this study, both pulsed-light-accelerated CXL protocols showed a significant improvement on CDVA, SE, topographic astigmatism, $\mathrm{K}_{\min }, \mathrm{K}_{\max }$ and $\mathrm{K}_{\mathrm{m}}$ at a 12-month follow-up without a between-group significant difference at any time point. Similar results in functional and topographic outcomes have been observed for the pulsed-light-accelerated CXL with $30 \mathrm{~mW} / \mathrm{cm}^{2}$ regardless the depth of the DL. ${ }^{4,11,23}$ Although Zhang et $\mathrm{al}^{26}$ reported a significant improvement in UDVA and CDVA in their epi-on pulsed-light $45 \mathrm{~mW} / \mathrm{cm}^{2}$ CXL protocol, they did not observe any significant improvement in $\mathrm{SE}, \mathrm{K}_{\max }$ or topographic astigmatism at a 12-month follow-up. Other than a longer follow-up to assess ulterior topographic changes for the latter study, a possible explanation of the lessen effect of CXL on corneal topography in this study is the use of a transepithelial CXL, since most of the available evidence favors epi-off CXL as the more effective method for strengthening the cornea and stabilizing keratoconus. $^{19,28,29}$ In our study, similar topographic and visual results than the A-CXL $(30 * 8)$ group were observed for the higher irradiance A-CXL $\left(45^{*} 5: 20\right)$ group despite the shallower DL. Group A-CXL $(45 * 5: 20)$ showed a significant improvement in UDVA since month 6 postoperative and until the end of the follow-up period, and although similar, group A-CXL's (30*8) improvement in UDVA was not statistically significant through the follow-up period. Also, significant improvement compared to baseline measurements in CDVA, SE, topographic astigmatism, $\mathrm{K}_{\min }, \mathrm{K}_{\max }$ and $\mathrm{K}_{\mathrm{m}}$ were observed in both groups at the end of the follow-up. Particularly, the decrease $>1.5 \mathrm{D}$ in $\mathrm{K}_{\min }, \mathrm{K}_{\max }$ and $\mathrm{K}_{\mathrm{m}}$ (Tables 2 and 3) at 12 months observed for $30 \mathrm{~mW} / \mathrm{cm}^{2}$ was noteworthy when compared to the modest decrease in keratometric parameters observed in similar reports., 41,25 Even when Bowes et $\mathrm{al}^{30}$ reported a similar behavior to our cohort in keratometries (decrease of $1.2 \mathrm{D}$ in $\mathrm{K}_{\max }$ at 12 
months, range $-5.6-+1.6$ D) using a pulsed-light-accelerated CXL protocol $\left(30 \mathrm{~mW} / \mathrm{cm}^{2}\right)$, evidence with regard to high irradiance pulsed-light protocols refractive, topographic and visual outcomes are scarce and inconclusive. Another consideration about the reported topographic result in our study is the young age of the patients; our cohort included 17 patients aged 19 or younger (mean age $19.76 \pm 4.63$ ), and as reported in previous experience with CXL in pediatric population, a different behavior is expected in the postoperative topographic profile (changes in $\mathrm{K}_{\max }$, Average K). ${ }^{31,32}$ With regard to the treatment failure rate, although not statistically significant difference was observed between groups, 3 $(13.5 \%)$ eyes in the group A-CXL $(30 * 8)$ and 2 eyes $(7 \%)$ in the A-CXL $\left(45^{*} 5: 20\right)$ group showed topographic progression of keratoconus, suggesting that our sample size was possibly inadequate to detect the effect size (change in $\left.\mathrm{K}_{\max }\right)$ with our specified study power $(\beta=0.20)$; hence, a larger sample size is necessary to detect statistically significant differences in topographic progression between groups.

None of the groups showed significant changes on ECD or complications associated to the CXL procedure at any point of the follow-up. Remarkably, both groups exhibited a significant decrease in CCT and TCT at month 1 postoperative, but both returned gradually over time to values similar to baseline. Greenstein et al described similar findings in 54 eyes with keratoconus after conventional CXL, with a significant thinning at month 1 postoperative and a progressive recovery to baseline measurements at month $12 .{ }^{33}$ In this matter, we described a moderate positive correlation between baseline CCT and the change in $\mathrm{K}_{\text {min }}, \mathrm{K}_{\max }$ and $\mathrm{K}_{\mathrm{m}}$, observing a more significant flattening in thinner corneas in both groups. A similar finding was described by Toprak et al who observed that corneas with baseline TCT less than $450 \mu \mathrm{m}$ were found significantly associated with more flattening in maximum keratometry.34 The reason and implications of these findings, although attributed to inherent biomechanical properties of thinner corneas, remain to be elucidated.

To our knowledge, this is the first report on the use of epi-off pulsed-light $45 \mathrm{~mW} / \mathrm{cm}^{2}$ irradiance CXL protocol. Although ex vivo evidence had shown that Bunsen-Roscoe reciprocity law was valid for irradiance values up to approximately $40-45 \mathrm{~mW} / \mathrm{cm}^{2,35}$ limited clinical data are available on the effectiveness of high irradiance CXL protocols due to the possible reduced biomechanical effect of CXL secondary to the increased oxygen consumption in the corneal stroma. ${ }^{36}$ Recently, an animal model assessing different CXL continuous irradiations between 3 and 90
$\mathrm{mW} / \mathrm{cm}^{2}$ with different exposure durations showed a consistent decrease in the stiffening of the tissue with reducing the irradiance duration (increasing irradiation). ${ }^{37}$ In this matter, pulsed UVA light is likely to have a compensatory effect to the oxygen depletion associated to the use of high levels of irradiance, optimizing intraoperative oxygen availability and increasing the effective biochemical crosslinking reactions in the corneal stroma, evidenced in many series as a deeper DL and stabilization or improvement in topographic and visual outcomes. ${ }^{10-12,23}$

This study is retrospective and comparative in nature (hence, it was not blinded or randomized), has short followup (12 months) and lacks a control group undergoing conventional (Dresden protocol) CXL, all of which may increase the risk of bias. Also, the study included measurements obtained from both eyes (right and left eyes) of the same subject and these data are usually correlated; therefore, the variance between eyes is usually less than that between subjects. In this matter, we decided to use both eyes in the statistical analysis to avoid rejecting valid data, reducing the potential power of the study and elude ethical considerations (subjecting patients to measurements that were not used in subsequent analysis). Finally, although no statistical difference was observed between the topographic and visual outcomes of the two aCXL protocols $(30 * 8$ vs $45 * 5: 20)$ and both protocols suggest being safe and effective to stabilize keratoconus progression at 12 months, increasing the power of the study (larger sample) is compulsory to ascertain these findings.

\section{Conclusion}

In conclusion, even when evidence has shown that topographic and functional improvement of keratoconus after CXL does not necessarily follow a linear trend over time due to stromal remodeling (especially in pediatric population) and a longer follow-up is needed to assess stabilization and regression, this study showed that for these two pulsed A-CXL protocols at a 12-month follow-up the hypothesis of a correlation between the DL depth and postoperative visual and topographic outcomes does not apply. Regardless of the DL depth, both protocols $(30 * 8$ and $45 * 5: 20)$ seem to be safe and effective to achieve topographic stabilization or improvement in $>85 \%$ of the eyes at 12 months; however, further studies with a longer follow-up and larger samples are mandatory to confirm these latest observations.

\section{Disclosure}

The authors report no conflicts of interest in this work. 


\section{References}

1. Raiskup-Wolf F, Hoyer A, Spoerl E, Pillunat LE. Collagen crosslinking with riboflavin and ultraviolet-A light in keratoconus: longterm results. J Cataract Refract Surg. 2008;34(5):796-801. doi:10.1016/j.jcrs.2007.12.039

2. Hersh PS, Greenstein SA, Fry KL. Corneal collagen crosslinking for keratoconus and corneal ectasia: one-year results. J Cataract Refract Surg. 2011;37(1):149-160. doi:10.1016/j.jcrs.2010.07.030

3. Asri D, Touboul D, Fournié P, et al. Corneal collagen crosslinking in progressive keratoconus: multicenter results from the French national reference center for keratoconus. J Cataract Refract Surg. 2011;37 (12):2137-2143. doi:10.1016/j.jcrs.2011.08.026

4. Toker E, Çerman E, Özcan DÖ, Seferoğlu ÖB. Efficacy of different accelerated corneal crosslinking protocols for progressive keratoconus. J Cataract Refract Surg. 2017;43(8):1089-1099. doi:10.1016/j. jcrs.2017.05.036

5. Wollensak G, Spoerl E, Seiler T. Riboflavin/ultraviolet-A-induced collagen crosslinking for the treatment of keratoconus. $\mathrm{Am} \mathrm{J}$ Ophthalmol. 2003;135(5):620-627. Available from: http://www. ncbi.nlm.nih.gov/pubmed/12719068. Accessed August 23, 2015.

6. Wollensak G. Crosslinking treatment of progressive keratoconus: new hope. Curr Opin Ophthalmol. 2006;17(4):356-360. doi:10.1097/01. icu.0000233954.86723.25

7. Ng AL, Chan TC, Cheng AC. Conventional versus accelerated corneal collagen cross-linking in the treatment of keratoconus. Clin Exp Ophthalmol. 2016;44(1):8-14. doi:10.1111/ceo.12571

8. Marino GK, Torricelli AAM, Giacomin N, Santhiago MR, Espindola $\mathrm{R}$, Netto MV. Accelerated corneal collagen cross-linking for postoperative LASIK ectasia: two-year outcomes. $J$ Refract Surg. 2015;31(6):380-384. doi:10.3928/1081597X-20150521-04

9. Alnawaiseh M, Rosentreter A, Böhm MRR, Eveslage M, Eter N, Zumhagen L. Accelerated $\left(18 \mathrm{~mW} / \mathrm{cm}^{2}\right)$ corneal collagen cross-linking for progressive keratoconus. Cornea. 2015;34(11):1427-1431. doi:10.1097/ICO.0000000000000578

10. Mazzotta C, Traversi C, Caragiuli S, Rechichi M. Pulsed vs continuous light accelerated corneal collagen crosslinking: in vivo qualitative investigation by confocal microscopy and corneal OCT. Eye. 2014;28(10):1179-1183. doi:10.1038/eye.2014.163

11. Moramarco A, Iovieno A, Sartori A, Fontana L. Corneal stromal demarcation line after accelerated crosslinking using continuous and pulsed light. J Cataract Refract Surg. 2015;41(11):2546-2551. doi:10.1016/j.jcrs.2015.04.033

12. Zhu Y, Reinach PS, Zhu H, et al. Continuous-light versus pulsedlight accelerated corneal crosslinking with ultraviolet-A and riboflavin. J Cataract Refract Surg. 2018;44(3):382-389. doi:10.1016/j. jers.2017.12.028

13. Seiler T, Hafezi F. Corneal cross-linking-induced stromal demarcation line. Cornea. 2006;25(9):1057-1059. doi:10.1097/01.ico.0000225720. 38748.58

14. Mazzotta C, Traversi C, Baiocchi S, et al. Corneal healing after riboflavin ultraviolet-A collagen cross-linking determined by confocal laser scanning microscopy in vivo: early and late modifications. $\mathrm{Am} \mathrm{J}$ Ophthalmol. 2008;146(4):527-533.e1. doi:10.1016/j.ajo.2008.05.042

15. Kymionis GD, Tsoulnaras KI, Grentzelos MA, et al. Evaluation of corneal stromal demarcation line depth following standard and a modified-accelerated collagen cross-linking protocol. $\mathrm{Am} J$ Ophthalmol. 2014;158(4):671-675.e1. doi:10.1016/j.ajo.2014.07.005

16. Bouheraoua N, Jouve L, El Sanharawi M, et al. Optical coherence tomography and confocal microscopy following three different protocols of corneal collagen-crosslinking in keratoconus. Invest Ophthalmol Vis Sci. 2014;55(11):7601-7609. doi:10.1167/iovs.14-15662

17. Shetty R, Pahuja NK, Nuijts RMMAMA, et al. Current protocols of corneal collagen cross-linking: visual, refractive, and tomographic outcomes. Am J Ophthalmol. 2015;160(2):243-249. doi:10.1016/j. ajo.2015.05.019
18. Gomes JAP, Tan D, Rapuano CJ, et al. Global consensus on keratoconus and ectatic diseases. Cornea. 2015;34(4):359-369. doi:10.1097/ ICO.0000000000000408

19. Soeters N, Wisse RPL, Godefrooij DA, Imhof SM, Tahzib NG. Transepithelial versus epithelium-off corneal cross-linking for the treatment of progressive keratoconus: a randomized controlled trial. Am J Ophthalmol. 2015;159(5):821-828.e3. doi:10.1016/j.ajo.201 5.02 .005

20. Mazzotta C, Balestrazzi A, Traversi C, et al. Treatment of progressive keratoconus by riboflavin-UVA-induced cross-linking of corneal collagen: ultrastructural analysis by Heidelberg retinal tomograph II in vivo confocal microscopy in humans. Cornea. 2007;26(4):390-397. doi:10.1097/ICO.0b013e318030df5a

21. Mazzotta C, Baiocchi S, Bagaglia SA, Fruschelli M, Meduri A, Rechichi M. Accelerated $15 \mathrm{~mW}$ pulsed-light crosslinking to treat progressive keratoconus: two-year clinical results. $J$ Cataract Refract Surg. 2017;43(8):1081-1088. doi:10.1016/j.jcrs.2017.05.030

22. Spadea L, Tonti E, Vingolo E. Corneal stromal demarcation line after collagen cross-linking in corneal ectatic diseases: a review of the literature. Clin Ophthalmol. 2016;10:1803-1810. doi:10.2147/OPTH. S117372

23. Peyman A, Nouralishahi A, Hafezi F, Kling S, Peyman M. Stromal demarcation line in pulsed versus continuous light accelerated corneal cross-linking for keratoconus. $J$ Refract Surg. 2016;32(3):206208. doi:10.3928/1081597X-20160204-03

24. Jiang L, Jiang W, Qiu S. Conventional vs. pulsed-light accelerated corneal collagen cross-linking for the treatment of progressive keratoconus: 12-month results from a prospective study. Exp Ther Med. 2017;14(5):4238-4244. doi:10.3892/etm.2017.5031

25. Mita M, Waring IVGO, Tomita M, Waring GO, Tomita M. Highirradiance accelerated collagen crosslinking for the treatment of keratoconus: six-month results. J Cataract Refract Surg. 2014;40 (6):1032-1040. doi:10.1016/j.jcrs.2013.12.014

26. Zhang X, Sun L, Chen Y, Li M, Tian M, Zhou X. One-year outcomes of pachymetry and epithelium thicknesses after accelerated $(45 \mathrm{~mW} /$ $\mathrm{cm}^{2}$ ) transepithelial corneal collagen cross-linking for keratoconus patients. Sci Rep. 2016;6(1):32692. doi:10.1038/srep32692

27. Pircher N, Lammer J, Holzer S, et al. Correlation between central stromal demarcation line depth and changes in $\mathrm{K}$ values after corneal cross-linking (CXL). Graefes Arch Clin Exp Ophthalmol. 2018;256 (4):759-764. doi:10.1007/s00417-018-3922-z

28. Raiskup F, Veliká V, Veselá M, Spörl E. Hornhautvernetzung beim Keratokonus: "Epi-off" oder "Epi-on"? Klin Monbl Augenheilkd. 2015;232(12):1392-1396. doi:10.1055/s-0035-1546155

29. Çerman E, Toker E, Ozarslan Ozcan D. Transepithelial versus epithelium-off crosslinking in adults with progressive keratoconus. $J$ Cataract Refract Surg. 2015;41(7):1416-1425. doi:10.1016/j.jcrs.2014.10.041

30. Bowes O, Coutts S, Ismailjee A, et al. Pulsed light accelerated corneal collagen cross-linking: 1-year results. Cornea. 2017;36(6): e15-e16. doi:10.1097/ICO.0000000000001187

31. Perez-Straziota C, Gaster RN, Rabinowitz YS. Corneal cross-linking for pediatric keratoconus review. Cornea. 2018;37(6):802-809. doi:10.1097/ICO.0000000000001579

32. Ulusoy DM, Göktaş E, Duru N, et al. Accelerated corneal crosslinking for treatment of progressive keratoconus in pediatric patients. Eur J Ophthalmol. 2017;27(3):319-325. doi:10.5301/ejo.5000848

33. Greenstein SA, Shah VP, Fry KL, Hersh PS. Corneal thickness changes after corneal collagen crosslinking for keratoconus and corneal ectasia: one-year results. J Cataract Refract Surg. 2011;37 (4):691-700. doi:10.1016/j.jcrs.2010.10.052

34. Toprak I, Yaylalı V, Yildirim C. Factors affecting outcomes of corneal collagen crosslinking treatment. Eye. 2014;28(1):41-46. doi:10.1038/ eye.2013.224

35. Wernli J, Schumacher S, Spoerl E, Mrochen M. The efficacy of corneal cross-linking shows a sudden decrease with very high intensity UV light and short treatment time. Invest Ophthalmol Vis Sci. 2013;54(2):1176. doi:10.1167/iovs.12-11409 
36. Hammer A, Richoz O, Mosquera SA, Tabibian D, Hoogewoud F, Hafezi F. Corneal biomechanical properties at different corneal crosslinking (CXL) irradiances. Invest Ophthalmol Vis Sci. 2014;55 (5):2881. doi:10.1167/iovs.13-13748
37. Bao F, Zheng Y, Liu C, et al. Changes in corneal biomechanical properties with different corneal cross-linking irradiances. $J$ Refract Surg. 2018;34(1):51-58. doi:10.3928/1081597X-20171025-01

\section{Publish your work in this journal}

Clinical Ophthalmology is an international, peer-reviewed journal covering all subspecialties within ophthalmology. Key topics include: Optometry; Visual science; Pharmacology and drug therapy in eye diseases; Basic Sciences; Primary and Secondary eye care; Patient Safety and Quality of Care Improvements. This journal is indexed on PubMed

Submit your manuscript here: https://www.dovepress.com/clinical-ophthalmology-journal
Central and CAS, and is the official journal of The Society of Clinical Ophthalmology (SCO). The manuscript management system is completely online and includes a very quick and fair peer-review system, which is all easy to use. Visit http://www.dovepress.com/ testimonials.php to read real quotes from published authors. 